Vargas L. y cols.

Rev. Chil. Pediatr. 66 (2); 98-102, 1995

\title{
Manifestaciones osteoarticulares en la presentación inicial de la leucemia linfoblástica aguda del niño
}

\author{
Lautaro Vargas P.1. 2; Marta Miranda M.1,
}

Bone and joint pain in patients with acute lymphocytic leukemia

\begin{abstract}
Bone and joiril signs and symploms were retrospectively studied in 70 consecutive patients under 15 years of age with ocute lymphocytic leukemia. Bone and or joint symproms, mainly pain, were recorded omong $37.5 \%$ of patients. Bone or joint poin were the primary couse of consulation in $17.4 \%$ of the whole cases, they were an accompanying, even though severe. spontoneously refered symptom in $12.9 \%$ of polienls and a mild event in onother $11.4 \%$ of affected children. The early hemaiclogical findings were nearly norral in the ritsi subgroup of patients and this accounled for initial mistaken anc delayed diagnosis, which included theumatic, arthapedic and even psychiaric conditons. In the other two subgroups there were no diagnostic errors in the first opproach. Osseous and arlicular findings did not modify prognosis, because there were nol significant differences in survival rates ornong patients with or without bone and or joint pain. Unexplained bone or joint pain together with minor hemaclocical abnormalities should prompl differential diagnosis with acule leukemia.
\end{abstract}

(Key words: leukemia, lymphocylic acute, arthritis, bone pain, osteoarhritis.)

Las manifestaciones osteoarticulares ocurren como síntoma inicial de la leucemia linfoblástica aguda (LLA) con mucha frecuencia. En algunos pacientes pueden ser de gran intensidad, hasta el punto de constituir el motivo de consulta. La relevancia de dichos síntomas puede sugerir diagnósticos errados, como afecciones traumatológicas, ortopédicas o reumatológicas. Algunos pacientes reciben tratamiento con antiinflamatorios no esteroidales (AINE) o corticoides que enmascaran la leucemia o aminoran los síntomas y signos hematologicos, que en muchos casos pueden ser leves o moderados ${ }^{l}$, lo que hace más difícil el diagnóstico definitivo. E] propósito del estudio que se expone fue determinar la incidencia de síntomas osteoarticulares como manifestación inicial en pacientes con leucemia linfoblástica aguda - especialmente cuando son el motivo de consulta-, así como también si su presencia, al retrasar el diagnóstico, constituye un factor negativo en el pronostico de estos enfermos.

1. Servicio de Pediatria, Hospital San Juan de Dios. Servicio de Salud Metropolitano Occidente.

2. Departamento de Pediatria Campus Occidente. Facultad de Medicina, Unjversidad de Chile.

\section{Material y Método}

Se analizaron retrospectivamente 70 niños menores de 15 años afectados de leucemia linfoblástica aguda, tratados en la unidad de hematología y oncología pediátrica del hospital San Juan de Dios, de Sanliago de Chile, en el transcurso de los años 1982 a 1991 . Se registró la presencia de dolor osteoarticular, su localización, duración, momento de aparición e intensidad. Los pacientes se clasificaron en tres grupos según si el dolor era el síntoma principal, acompañante pero relatado espontáneamente o leve y era necesario el interrogatorio dirigido para obtener el antecedente. En los pacientes en que los dolores osteoarticulares eran el motivo primario de consulta, el síntoma podia ser difuso o localizado, en las metafisis o las diáfisis de los huesos largos o en grandes y pequeñas articulaciones, pero intensa, con impotencia funcional, claudicación. irritabjlidad, inapetencia, Janto o insomnio y los afectados requerían analgésicos o antiinflamatorios no esteroidales (AINE) en forma permanente. En el segundo grupo el dolor era un sintoma referido espontáneamente en la historia, pero siempre acompanando a otras manifestaciones, especialmente de la esfera hematológica, que eran el principal motivo de preocupación de los padres; la impotencia funcional - cuando estaba presente- era pasajera o de menor cuantía y usaban analgésicos de manera intertmitente. En la tercera categorfa los pacientes sufrian síntomas osteoarticulares leves o moderados, ocasionales, que en muchos casos no fueron referidos espontáneamente por los padres, detectándose por preguntas dirigidas y no siempre requerían de analgésicos o AINE. Además se analizó la duración del síntoma dolor desde su aparición hasta el momento del 
diagnostico de leucemia linfoblástica aguda, Jos diagnósti. cos formulados previamente y los signos bematologicos detectados en el momento del diagnóstico. Se calculo la curva de sobrevida del colal de los pacientes y de los que presentaban espontáneamente manifestaciones osteoarticulares. No se analizaron radiografias osteoarticulares por. que en muchos casos no se hicieron.

\section{Resultados}

La frecuencia de manifestaciones dolorosas osteoarticulares fue $35,7 \%$ ( 25 de 70 casos). El dolor fue intenso y causa de la consulta en 8 $(11,4 \%)$ niños con leucernia linfoblástica, síntoma acompañante de importancia en $9(12,9 \%)$ de ellos y leve a moderado en otros $8(11,4 \%)$ casos, en proporciones prácticamente iguales en los tres subgrupos.

En todos los enfermos cuyas manifestaciones osteoarticulares fueron el síntoma principal y motivo de consulta, se hizo gran variedad de diagnósticos reumatológicos, ortopédicos y traumatológicos, que incluyeron enfermedad reumática $(\mathbf{n}=2)$, artritis reumatoidea o reactiva, coxalgia, osteomielitis, artritis piogena o sinovitis transitoria, enfermedad de Perthes, dolores sicosomáticos o sinovitis transitoria $(\mathbf{n}=\mathbf{1}$ de cada uno). Varios casos fueron tratados con corticoides o AINE (los más usados fueron ácido acetilsalićlico e indometacina). La duración de los dolores varió entre 4 y 14 días en 15 casos $(60 \%)$, entre 2 y 4 semanas en 7 pacientes $(28 \%)$ y entre 1 y 8 meses en tres casos (12\%). En los ocho niños en que el dolor osteoarticular fue el motivo de consulta, este duro mas de tres semanas y causó entre dos y once consultas antes del diagnóstico. En muchos de estos pacientes se realizaron hemogramas que no mostraron alteraciones importantes; sólo al persistir los síntomas y repetir el hemograma se encontraron leves alteraciones, si bien sugerentes de leucemia, que indujeron a derivarlos a hematología. Algunos pacientes se manifestaron sólo como artralgias, otros poliartritis y otros con dolores óseos diafisiarios y metafisiarios.

En las tablas 1 y 2 se describen las características hematologicas en el momento del diagnóstico definitivo de los enfermos en que los síntomas osteoarticulares eran el motivo de consulta o constituían un acompañante relevante. En muchos de éstos se habían realizado hemogramas previos que sólo mostraban leves alteraciones, principalmente anemia, pero éstas más el antecedente de dolores por tiempo prolongado sin un diagnóstico preciso permitieron sospechar leucemia. Esta se confirmó con el mielograma que mostró infiltración blástica en más de $80 \%$ de los casos. En algunos pacientes los estudios cintigráficos óseos sugerían infiltración neoplásica y no afección inflamatoria, lo que motivó su derivación a hematología. En un caso de dolor como motivo de consulta el hemograma y el examen físico eran normales y otro enfermo s6lo tenía discreta anemia y leve disminución de plaquetas, pero con hepatomegalia.

En los pacientes con síntomas osteoarticulares significativos como acompañantes también se observan alteraciones hematológicas dis-

Tabla 1

Aspectos hematológicos y clínicos al momento del diagnóstico de leucemia linfoblástica aguda en pacientes con dolores osteoarticulares como motivo principal de consulta

\begin{tabular}{|c|c|c|c|c|c|c|c|}
\hline Sexo & $\begin{array}{c}\text { Edad } \\
\text { (a) }\end{array}$ & $\begin{array}{l}\text { Hto } \\
(\%)\end{array}$ & $\begin{array}{l}\text { Leucoct } \\
\left.(\mathrm{x}] 0^{3} / \mu \mathrm{l}\right)\end{array}$ & $\begin{array}{c}\text { Blastos } \\
\%\end{array}$ & $\underset{\left(x 10^{3} / \mu \mathrm{l}\right)}{\text { Plaqt }}$ & $\begin{array}{c}\text { Hígado } \\
\mathrm{cm}\end{array}$ & $\begin{array}{c}\text { Bazo } \\
\mathrm{cm}\end{array}$ \\
\hline F & 5 & 40 & 5.2 & 0 & 200 & - & - \\
\hline $\mathrm{F}$ & 13 & 31 & 5,7 & 15 & 85 & - & - \\
\hline $\mathbf{M}$ & 12 & 18 & 1,3 & 9 & 9 & 2 & - \\
\hline$F$ & 5 & 30 & 8,3 & 3 & 80 & - & - \\
\hline $\mathbf{F}$ & 8 & 34 & 15,0 & 0 & 125 & 3 & - \\
\hline M & 3 & 27 & 2,5 & 5 & 30 & - & 1 \\
\hline $\mathbf{M}$ & 5 & 15 & 3,6 & 30 & 15 & 2 & - \\
\hline $\mathrm{M}$ & 5 & 29 & 4,8 & 10 & 168 & 2 & - \\
\hline
\end{tabular}

Hto:hemalocrito. Leucoct:leucocitos. Plaqt:plaquetas.

F: femenino. M: masculino. 
Tabla 2

Aspectos hematológicos y clfricos en el momento del diagnóstico de leucemia aguda linfoblástica con dolor osteoarticular como síntoma acompañante

\begin{tabular}{|c|c|c|c|c|c|c|c|}
\hline Sexo & $\begin{array}{l}\text { Edad } \\
\text { (a) }\end{array}$ & $\begin{array}{l}\text { Hto } \\
(\%)\end{array}$ & $\begin{array}{l}\text { Leucoct } \\
\left(x \mid 0^{3} / \mu \mathrm{J}\right)\end{array}$ & $\begin{array}{c}\text { Blastos } \\
\%\end{array}$ & $\begin{array}{c}\text { Plag1 } \\
\left(\times 10^{3} / \mu \mathrm{J}\right)\end{array}$ & $\begin{array}{c}\text { Hígado } \\
\text { cm }\end{array}$ & $\begin{array}{c}\text { Bazo } \\
\text { cm }\end{array}$ \\
\hline$M$ & 5 & 28 & 4,1 & 46 & 2 & - & - \\
\hline $\mathrm{F}$ & 2 & 12 & 5,5 & 0 & 26 & 3 & - \\
\hline$M$ & 5 & 17 & 234,0 & 99 & 10 & 6 & 10 \\
\hline$M$ & 5 & 27 & 15,3 & 50 & 88 & 2 & - \\
\hline M & 5 & 33 & 11,0 & 58 & 70 & - & - \\
\hline M & 13 & 30 & 5,8 & 14 & 15 & - & - \\
\hline M & 15 & 37 & 13,4 & 51 & 2 & 4 & 4 \\
\hline M & 2 & 37 & 3,2 & 3 & 5 & - & - \\
\hline$M$ & 2 & 19 & 6,5 & 59 & 17.5 & 4 & - \\
\hline
\end{tabular}

Hto:hematocrito. Leucoct:leucocitos. Plaql:plaquetas.

F: femenino. M: masculiso.

cretas o moderadas, pero suficientes para sospechar el diagnóstico de leucemia. En un solo caso no se encontraron blastos circulantes, pero había anemia y trombocitopenia importantes. En el tercer grupo prácticamente todos presentaban un aspecto claro de insuficiencia medular, así como también la mayoría tenía visceromegalia $o$ adenopatías. Los enfermos incluidos fueron sometidos a diferentes protocolos de tratamiento debido a que la muestra comprende un período de 10 años de observación. La sobrevida libre de eventos a 10 años, según tabla de Kaplan Meir, con mediana de 5 anios, fue $54 \%$ en el total de los pacientes, $61 \%$ para los que sufrían manifestaciones osteoarticulares y $52 \%$ en los niños sin dolor. No hay diferencia estadísticamente significativa entre los grupos con y sin manifestaciones dolorosas.

\section{Comentario}

Las manifestaciones osteoarticulares son frecuentes en las afecciones neoplásicas del niño, aun en las que no afectan primariamente los huesos o las articulaciones ${ }^{1-6}$. La afección osteoarticular es muy variable, puede presentarse desde comienzo o en otro momento del curso de la enfermedad, en forma primaria (como en los tumores óseos) o por extensión a distancia (metástasis ósea) o una complicación del tratamiento. En pediatría es más raro que las manifestaciones osteoarticulares formen parte de un síndrome paraneoplásico?
En la leucemia aguda linfoblástica existe, por definición, compromiso difuso de la médula ósea. Esto explica la alta frecuencia de afección ósea en casi todas las necropsias, aun si las radiografías no muestran lesiones ${ }^{8}$. La expresión de ella incluye osteopenia, alteraciones metafisiarias, osteolisis, periostitis, osteoesclerosis $y$ alteraciones articulares ${ }^{9 .}{ }^{10}$. En los niños las lesiones más frecuentes son las bandas radiolúcidas horizontales y las líneas de detención del crecimiento que, aunque inespecíficas, son de gran ayuda en el diagnóstico, especialmente en los casos con pocas alteraciones en el hemograma. Su alta prevalencia en los niños y rareza en los adultos sugiere que su causa radica en interferencia con la osteogénesis más que en infiltración leucémica, la que habirualmente es escasa en el resto de la metáfisis ${ }^{9}$. Las lesiones osteolíticas pueden ser localizadas o múltiples. Generalmente obedecen a lesiones combinadas de necrosis, hemorragia, infartos oseos e infiltración leucémica ${ }^{9,11,12}$, que en algunos pacientes pueden ocasionar fracturas patológicas. La osteopenia difusa se puede deber a una alteración del metabolismo o ser secundaria a la infiltración leucémica, la que explica el ensanchamiento de la médula y el adelgazamiento de la cortical. Los esteroides empleados en el tratamiento también contribuyen a estas manifestaciones $^{3}$. Los infiltrados periostales y corticales son asimismo frecuentes en los niños y también producen dolor. Los infiltrados leućrnicos se extienden atraves de los canales de Havers has- 
ta el periostio, produciendo reabsorción del hueso circundante. El periostio puede ser levantado por el infiltrado o por hueso de neoformación. Las manifestaciones articulares pueden ser monoarticulares, a veces son migratorias, afectando especialmente a las rodillas $y$ se deben principalmente a hemorragia en $O$ alrededor de las articulaciones con elevación del periostio periarticular ${ }^{9}$ 10. En general la infiltración leucémica no es muy notoria en estos territorios.

La frecuencia de síntomas osteoarticulares que se describe en esta serie es mayor que la descrita anteriormente en la misma unidad clíni$\mathrm{ca}^{5}$, lo que puede deberse a que, más recientemente, se buscan estos síntomas con mayor acuciosidad y se complementa el estudio con evaluación por reumatología. La frecuencia de dolores osteoarticulares en la leucemia varía, en otras experiencias, entre 23 y $40 \%^{1-6}$.

Los problemas de diagnóstico diferencial se produjeron en $11,4 \%$ de estos casos, que corresponden a los que tuvieron síntomas osteoarticulares de gran magnitud. La variecad de diagnósticos propuestos se relaciona con el carácter proteiforme de estas manifestaciones, a veces dolores óseos localizados o difusos, en otras oportunidades artralgias o artritis, de ubicación y curso cambiantes. Jonsson registró este tipo de enfermos en $18 \%$ de sus casos. También en el informe que se comenta, como en atros ${ }^{8}$ 13 $^{1}$ los pacientes con intensos sintomas osteoarticulares suelen mostrar pocas alteraciones en los exámenes hematologicos, lo que explica el retraso del diagnóstico. Como en otros reportes, tampoco encontramos diferencias significativas en la sobrevidal.

$\mathrm{El}$ análisis acucioso de los síntomas osteoarticulares permite establecer diferencias semiologicas con los patrones clásicos de las enfermedades reumatológicas y ortopédicas con que se confunden inicialmente estos enfermos ${ }^{14}$, 15. En todo niño con síntomas o signos osteoarticulares -artralgias, artritis o dolor óseo- se debe plantear el diagnóstico diferencial con leucemia, en especial si ellos difieren de los clásicos de otras afecciones óseas o articulares y se acompañan de alteraciones aisladas o combinadas de las series sanguíneas, aun cuando éstas sean leves. El examen físico meticuloso, complementado con estudio de imágenes y de laboratorio, incluido un mielograma, permitirán proponer el diagnós- tico correcto de leucemia en etapas previas a la expresion de sus manifestaciones habituales.

\section{Resumen}

Se describen retrospectivamente las manifestaciones óseas y articulares de 70 pacientes de leucemia linfoblástica aguda, menores de 15 años, atendidos a lo largo de 9 años (1982 a 1991). Se registró dolor osteoarticular en $37,5 \%$ de ellos, en $8(11,4 \%)$ eran muy intensos, ensombrecían los síntomas hematológicos y constituían el motivo de consulta; en $9(12,9 \%)$ el dolor era importante y relatado espontáneamente, pero era un acompañante de los síntomas hematológicos que motivaban la consulta; en otros $8(11,4 \%)$ casos el dolor era leve, ocasional o no referido espontáneamente. Las manifestacione hematologicas eran poco marcadas en los primeros, aun en el momento de identificar la leucemia, después de varias semanas de dolor osteoarticular, lo que explica los errores en los diagnósticos iniciales (enfermedad reumática, artritis reumatoidea, sinovitis transitoria, enfermedad de Perthes, osteomielitis o trastorno psiquiátrico) en todos estos pacientes y el retraso del diagnóstico de leucemia. En los niños cuyos dolores osteoarticulares eran significativos pero en carácter de acompañantes de otro síntoma, no hubo errores iniciales en el diagnóstico, enfocándose el estudio a una afección hematológica, ya que los sjgnos principales la sugerian. En los pacientes con poco dolor la situación era aún más clara. El dolor osteoarticular no modific 6 , sin embargo, el pronostico, pues la sobrevida en los pacientes que lo sufrían fue similar que en los sin dolor. Los síntomas osteoarticulares sin explicación clara, sumados a alguna alteración hematológica, obligan a incluir leucemia en el diagnóstico diferencial.

(Palabras clave: leucemia, linfocítica aguda, artritis, artralgia, dolor oseo, osteoartritis.)

\section{Referencias}

1. Jonsson $D G$. Sartain P, Ducare J, et al.: Bone pain as an inilial symptom of childhood acute lymphoblastic leukemia: association with nearly normal hematologic indexes. J Pediatr 1990; 117: 233-237.

2. Miller $D$ : Acute lymphoblastic leukemia. Pediatr Clin North Am 1980; 27: 269-291. 
3. Rostion C, Cerda I, Valdecantos E, Hernúndez $R$, Macdoo X: Dolor óseo en leucemia. Resumen XXVIl Jornadas Investigación Pediátrica Santiago Norte 1992. Pediatrí (Santiago) 1992; 35: 218-219.

4. Thomas Lu Farkner CE, Frei E, et al.: The skeletal lesions of acute leukemia. Cancer 1961; 14: 508-621.

5. Vargas L. Pino $S$, Barría M, Young $T$, García $H$ : Aspectos clínicos y heratológicos iniciales en la lewcemia aguda del niño. Rev Chil Pediatr 1984; 55 : 149 153.

6. Sallan $S$, Niemeyer $\mathrm{Ch}$ : Acute lymphoblastic leukemia. En Hematology of lnfancy and Childhood. Nathan D.G.,Oski F.A. (eds.). Pbiladelphia,W.B. Saunders 1993: 1249-1248.

7. Resnick D: Myeloproliferative disorders. En Diagnosis of bone and joint diseases. Resuick D, Niwayama (eds.). Philadelphia, W.B. Saunders 1981:1992-2016.

8. Revesz $T$, Kardos $G$, Kaytar $P$, et al.: The adverse effect of prolonged prenisonolone pretreatment in children with acute lymphoblastic leukemia. Cancer 1985: 55 ; 1637-1640.
9. Radríguez de la Serna A: Colagenosis paraneoplásicas. Rev Espaitola de Reumatología 1991; 18: 143-152.

10. Amromin GD: Skin and musculoskeletal system. En Pathology of Leukemia. Amromin G.D.(ed.) New York, Hoeber 1968; 309-346.

11. Silverstein Mn. Kelly P: Leukenia with osteoarticular symptoms and signs. Ann Inter Med 1963; 59: 637 645.

12. Marsh JR, Wyiund $D J$, Heath VC: Ostenarticular and pulmonary manifestations of acute leukemia: case report and review of the literature. Cancer 1986; 57 : 385-390.

13. Willson $I K$ : The bone lesions of childhood leukemia: a survey of 140 cases. Radiology 1959:72:672-681.

14. Pui $C$. Stass $S$, Green A: Bone marrow necrosis in children with malignant disease. Cancer 1985; 56: 1522-1525.

15. Nies BA, Kundel D. Thomas L: Leukopenia, bone pain, and bone necrosis in patienls with acute leukemia: a clinicopathologic complex. Ann Intern Med 1965; 65:698-705. 